\title{
In Situ Optimal Reshading of Arrays with Failed Elements
}

\author{
MICHAEL S. SHERRILL AND ROY L. STREIT, SENIOR MEMBER, IEEE
}

(Invited Paper)

\begin{abstract}
An algorithm is presented which computes optimal weights for arbitrary linear arrays. The application of this algorithm to in situ optimal reshading of arrays with failed elements is discussed. It is shown that optimal reshading can often regain the original sidelobe level by slightly increasing the mainlobe beamwidth. Three examples are presented to illustrate the algorithm's effectiveness. Hardware and software issues are discussed. Execution time for a 25-element array is typically between 1 and 2 min on an $\mathrm{HP9836C}$ microcomputer.
\end{abstract}

\section{INTRODUCTION}

A linear array of discrete elements (sensors) often experiences element failures in situ. These failures can significantly increase the sidelobe levels of the array wavenumber response, depending on how many elements fail and where the elements are located within the array. We discuss here an optimal reshading (reweighting) algorithm which can be applied in situ to reduce the sidelobe levels to the original design level. In many common element-failure situations, optimal reshading can regain the original sidelobe level by slightly increasing the mainlobe beamwidth. In arrays which experience significant element failures, optimal reshading is still possible, but may be of limited use. Three examples given below demonstrate a few of the possibilities.

An algorithm for optimal reshading was first proposed in [1] by Streit and Nuttall. Their algorithm utilized the generalpurpose subroutine [2] to solve a specially structured "linear programming" problem. Unfortunately, their algorithm required hours of computation time and large amounts of computer storage on a minicomputer (the VAX 11/780) to optimally reshade a 50 -element array with five failed elements. Consequently, their algorithm is not useful for in situ optimal reshading.

The shading algorithm proposed here differs from Streit and Nuttall's primarily in that we solve their linear programming problem using a new general-purpose subroutine [3], [4], herein referred to as Algorithm 635. Algorithm 635 uses the special structure of the linear programming problem to reduce time and storage requirements by orders of magnitude. Algorithm 635 can be incorporated easily in Streit and Nuttall's original approach. A significant algorithmic improvement was discovered in the course of this study and is described below. The resulting shading algorithm is fast enough and small enough to execute successfully on micro-

Manuscript received March 11, 1986; revised August 11, 1986.

The authors are with the Naval Underwater Systems Center, New London, CT 06320.

IEEE Log Number 8714258. computers (such as the HP9836C used here) in only a few minutes. Typical execution time for a 25 -element array is under $2 \mathrm{~min}$; for a 50 -element array, execution time is typically under $10 \mathrm{~min}$. The current algorithm, and the HP9836C with its inherent transportability, comprise an effective system for optimal reshading in situ.

\section{Optimal ARRay Shading}

The wavenumber response of a linear array composed of $N$ discrete omnidirectional elements located at arbitrary fixed positions $x_{n}$ is given by

$$
T(k)=\sum_{n=1}^{N} w_{n} \exp \left[-i k x_{n}\right]
$$

where $w_{n}$ are the element weights and the independent variable $k$ denotes wavenumber in radians per unit length. The element weights are required to be real, but this entails no loss of generality (see below in Section III). Also, from (1), $T(-k)$ $=T^{*}(k)$ for real weights (asterisk denotes conjugation), so it is unnecessary to consider negative values for $k$ and we confine our attention to nonnegative $k$.

The array response as a function of $k$ can be considered to be composed of a mainlobe beamwidth and a sidelobe region. The objective of the optimization process is to make $|T(k)|$ as small as possible on the user-specified sidelobe interval. Array weights which achieve this objective are said to be optimal. The optimization process usually produces equivalued sidelobes in the sidelobe region.

Weights that are optimal for a full array do not remain optimal after the array experiences element failures. To partially compensate for failed elements, the array is optimally reshaded by undertaking the optimization process again and incorporating knowledge of which elements have failed. As the examples below will show, the effectiveness of this strategy depends upon how many elements have failed and the location of these elements in the array.

The sidelobe interval is defined differently depending on the interelement spacing of the array. For an array with periodically spaced elements and no failures, the sidelobe interval is defined to be $\left[K_{0},(2 \pi / D)-K_{0}\right]$, where $K_{0}$ is calculated from the desired sidelobe level and the number $N$ of array elements. ${ }^{\prime} D$ is the physical distance from sensor to sensor.

\footnotetext{
${ }^{1}$ For an $N$-element array and $-t$-dB peak sidelobes, we have $K_{0}=(2 / D)$ $\arccos \left(1 / Z_{0}\right)$ where $2 Z_{0}=\left[r-\left(r^{2}-1\right)^{1 / 2}\right]^{1 / M}+\left[r+\left(r^{2}-1\right)^{1 / 2}\right]^{1 / M}$ $r=10^{r / 20}$, and $M=N-1$. The interelement spacing $D$ is assumed to be half of the so-called design wavenumber, and $N$ is the number of array elements before failures.
} 
Furthermore, the minimization interval can be reduced to $\left[K_{0}\right.$, $\pi / D$ ], since the response of this array is symmetric about $k=$ $\pi / D . K_{0}$ is typically the point on the mainlobe response which is equal in magnitude to the peak of the sidelobes, but this is not always true for seriously degraded and/or aperiodic arrays (see Example 3 below). For arrays with aperiodically spaced elements, the sidelobe-interval, denoted by $\left[K_{0}, K_{1}\right]$, must be chosen by inspection of a nonoptimal beam pattern or some other means. $|T(k)|$ must be minimized over the full $\left[K_{0}, K_{1}\right]$ range since, in general, an aperiodic array response is not symmetric about any wavenumber other than $k=0$. The ability to specify arbitrary $K_{0}$ and $K_{1}$ is particularly useful for those applications involving aperiodically spaced elements because lower sidelobe levels may be obtained by looking at different minimization regions.

The optimization process deals with element failures in an array in the following way.

Step 1. Maintain mainlobe beamwidth and permit the sidelobe levels to rise.

Step 2. Regain, if possible, the original sidelobe level by broadening the mainlobe.

Broadening the mainlobe by increasing $K_{0}$ (step 2) is performed only if the sidelobe level, even after optimal reshading, has risen to an unacceptable value because of element failures. Thus step 1 is normal algorithmic procedure, and step 2 requires some iteration in specifying $K_{0}$ and/or $K_{\mathrm{I}}$ because a compromise has to be made between the mainlobe beamwidth and the level of the sidelobes.

The solution of the array problem in the original formulation [1] is mathematically equivalent to solving an overdetermined system of complex linear equations. Unacceptably high sidelobes result if this system is solved in the usual least squares sense, so it is necessary to solve the system so that the magnitude of the maximum residual error is minimized. There now exists [3] an efficient algorithm and corresponding FORTRAN code [4] for solving problems of this sort to high accuracy.

To obtain the beamformer equation in an appropriate format to utilize this algorithm, we normalize the peak response of $T(k)$ so that $T(0)=1$. This gives

$$
\sum_{n=1}^{N} w_{n}=1
$$

We solve (2) for the $N$ th weight $w_{N}$ and substitute in (1) to obtain

$$
T(k)=\exp \left[-i k x_{N}\right]+\sum_{n=1}^{N-1} w_{n}\left[\exp \left(-i k x_{n}\right)-\exp \left(-i k x_{N}\right)\right]
$$

By sampling $T(k)$ at the $M$ equispaced points

$$
k_{m}=K_{0}+\frac{\left[K_{1}-K_{0}\right]}{M-1}(m-1), \quad m=1, \cdots, M
$$

we can write the problem of minimizing the peak sidelobe level of the array response as

$$
\min _{\left\{w_{n}\right\}} \max _{1 \leq m \leq M}\left|f_{m}-\sum_{n=1}^{N-1} a_{m n} w_{n}\right|
$$

where the complex numbers $f_{m}$ and $a_{m n}$ are defined by

$$
\begin{gathered}
f_{m}=\exp \left[-i k_{m} x_{N}\right] \\
a_{m n}=\exp \left[-i k_{m} x_{N}\right]-\exp \left[-i k_{m} x_{n}\right] .
\end{gathered}
$$

The problem (5) is precisely the form necessary for application of Algorithm 635. For theoretical details of this algorithm, the interested reader is referred to [3].

Sometimes a few of the optimum weights for arrays with failed elements are observed to be negative, particularly those on the end elements. If the weights are applied in hardware, providing a $180^{\circ}$ phase factor on the element output may not be desirable or possible. However, Algorithm 635 allows the selection of all nonnegative weights; this is implemented by the addition of constraints to (5). Usually, but not always, an element is zeroed if it would have had a negative weight. From (2) it follows that, if all the element weight values are required to be positive, they must be between 0 and 1 . The requirement that weights $w_{1}, \cdots, w_{N-1}$ be between 0 and 1 can be written mathematically as

$$
\left|w_{n}-\frac{1}{2}\right| \leq \frac{1}{2}, \quad n=1, \cdots, N-1 .
$$

Algorithm 635 requires these $N-1$ constraints. Algorithm 635 can also incorporate any number of general constraints of the form

$$
\left|\sum_{n} b_{m n} w_{n}-c_{m}\right| \leq d_{m}, \quad m=1,2, \cdots, L
$$

where $c_{m}$ and $d_{m}$ are constants. The requirement that $w_{N}$ also be nonnegative gives

$$
\left|\left(1-\sum_{n=1}^{N-1} w_{n}\right)-\frac{1}{2}\right| \leq \frac{1}{2}
$$

or

$$
\left|\sum_{n=1}^{N-1} w_{n}-\frac{1}{2}\right| \leq \frac{1}{2}
$$

which is clearly a special case of the general constraints (8).

\section{Algorithm Improvements}

Several changes to the algorithm presented in [1] enable significant reduction in the need for computational intensity. Lewis and Streit [5] have proved that, for a general line array shaded so that it has optimal sidelobe levels when steered through the same number of degrees either side of broadside, there exists a set of optimal weights that are real. Thus complex weights do not need to be considered. This fact allows an approximate eight-fold reduction in computation time and a two-fold reduction in storage requirements. 
It is clear that the 50-element example run in Streit and Nuttall [1] was significantly oversampled in wavenumber. Their beam pattern can be reproduced with a four-fold reduction in the sampling of $T(k)$ (see Example 2 below), and this in no way detracts from the practical application of the algorithm. A significant reduction in computation time is realized by decreasing the number $M$ of beam pattern samples in (4).

A significant algorithmic modification made to Algorithm 635 further decreases computation time. We have labeled this modification "fast costing" and it is an important step in making the algorithm feasible on microcomputers such as the HP9836C. In order to describe this modification properly, some familiarity with the simplex method of linear programming and reference [3] is assumed.

Algorithm 635 can be broken into two fundamental computational operations called "costing" and "pivoting." "Costing" determines the so-called minimum reduced cost coefficient and requires $2 N M$ multiplications, where $N$ is the number of discrete array elements and $M$ is the number of samples taken of the beam pattern. "Pivoting" is a basis update and requires $N^{2}$ real multiplications. It is clear that the speed of the algorithm is intimately related to the number $M$ of samples taken of the beam pattern, as well as the number $N$ of discrete array elements. Since $M$ is larger than $N$, "costing", requires more multiplications than "pivoting."

"Costing" in the linear array application means that, in each simplex iteration, the "discretized absolute value" of every sidelobe sample of the wavenumber response function $T\left(k_{m}\right)$, $m=1, \cdots, M$, is computed to determine the "minimum reduced cost coefficient" of the current "basic feasible solution." By proceeding through a finite sequence of such "basic feasible solutions," we arrive at the solution of the "discretized problem." As shown in [3], this implies that the computed optimal wavenumber response function can have sidelobe levels that are theoretically at most $0.04 \mathrm{~dB}$ higher than the true optimum sidelobe level. " "Fast costing" refers simply to the fact that we first determine which of the sidelobe samples $T\left(k_{m}\right), m=1, \cdots, M$, has the largest true absolute value, and then compute the "discretized absolute value" of this one complex number. Therefore, only one "discretized absolute value" calculation is performed in each simplex iteration instead of $M$ such calculations. The resulting reduction in computational effort is significant in microcomputing environments. The drawback is that the use of "fast costing" prevents the simplex algorithm from converging to a solution of the "discretized problem." Fortunately, however, it can be proved that we must approximate the solution in a well-defined sense. In the linear array application, "fast costing" results in the computed optimum beam pattern having sidelobe levels that are theoretically at most $0.08 \mathrm{~dB}$ higher than the true optimum level. ${ }^{3}$ This is a small price to pay for major execution time improvements.

\footnotetext{
2 The theoretical error of at most $0.04 \mathrm{~dB}$ is derived by taking $20 \log _{10}$ (sec $(\pi / p))$, where $p=32$. The term $\sec (\pi / p)$ is the error bound discussed in [3].

${ }^{3}$ Fast costing squares the error bound, giving $\sec ^{2}(\pi / p)$, or $0.08 \mathrm{~dB}$ when $p=32$.
}

\section{Algortthm Implementation for IN Situ Use}

An algorithm must be reliable, easy to use, and fast when executing on portable microcomputers, to be useful for in situ application. The following section details the most important hardware and software issues addressed to enable in situ optimal reshading of arrays with failed elements.

The algorithm has been coded in BASIC and is comprised of Algorithm 635 and an array processing driver program. Algorithm 635 solves the linear program for a set of optimal weights, given data supplied by the driver program. The driver performs the initial setup based on several user inputs and provides all program output.

The driver program may be used with linear arrays having either periodic or aperiodically spaced elements. Program output consists of a graph of the optimal beam pattern, a graph of the optimal normalized element weights, and several parameters pertinent to the specific problem. Provision is made for storing the weights in a separate data file for possible use with digital beamformers.

A Hewlett-Packard (HP) specific software modification was made by setting up the input data arrays (equation (6)) in buffers so that they are accessible for a one-dimensional multiply. For large-array dimensions, indexing a doubly subscripted data array and performing a dot product takes more time on the HP9836C than reading in a data array from a buffer, doing a MAT multiply, and performing a summation. (A MAT multiply is simply an element-by-element multiply of two equally dimensioned data arrays.) However, this procedure is more time consuming when the input data arrays are very small (i.e., the number of elements in the line array is small). The break-even point occurs at around 12 or 13 elements, so it was decided to incorporate this speed enhancement for the longer-running larger line arrays and trade off some speed reduction on the smaller line arrays.

To obtain fast execution times for in situ applications, we use one hardware speed enhancement, a $12.5-\mathrm{MHz}$ fast $\mathrm{CPU}$ card with 16 kbytes of cache memory. This hardware supplement is available from HP for use on the HP9836C. Cache memory is fast memory resident on the CPU card for quick instruction acquisition. The use of the fast CPU board rather than the 8- $\mathrm{MHz}$ clock present in the standard computer configuration results in an approximate factor-of-two increase in observed speed.

The complete program is precompiled by use of software and a floating point math card available from the INFOTEK company. Precompilation reduces most computational portions of the BASIC code to machine language, giving an additional three-fold reduction in computation time. It is also desirable to upgrade the operating system for the HP to its latest revision. All work on these problems was run using the BASIC 3.0 operating system and the hardware supplements noted above.

Computation time is defined as time spent in Algorithm 635 and does not include the small amount of set-up time required by the driver program. Computation times are for the compiled BASIC program run on the HP9836C with the special hardware additions mentioned above.

The program described here needs just over 303 kbytes of 
internal memory in addition to the memory required by the operating system to execute on the HP9836C. This is the amount of space required by fixing the maximum array size at $N=50$, and allowing at most $M=256$ beam pattern samples. Users can change dimensions to suit their specific needs, but storage requirements presently are directly proportional to the product $N M$. Even for a much larger number of line array elements, it is unlikely that memory restrictions would prove to be a problem on the HP9836C since extra memory boards of 1 Mbyte each are readily available.

Ongoing modifications should further enhance the capability and speed of the BASIC algorithm and driver. The addition of the ability to handle directional sensors is both useful and straightforward to implement. Execution of identical code on the new HP 300 series computers, which have a $16.6-\mathrm{MHz}$ clock rate, should further reduce the computation time. Computation times on the order of $5 \mathrm{~min}$ for a 50 -element array and $1 \mathrm{~min}$ for a 25 -element array are anticipated.

It is possible to run the BASIC program in its uncompiled state. The execution of the program with cache memory and the fast CPU board as the only enhancements results in computation times of approximately $25 \mathrm{~min}$ for a 50 -element array and $4.5 \mathrm{~min}$ for a 25 -element array.

A copy of the entire program is available from the authors. Our specific implementation in HP BASIC utilizes several hardware and software devices to achieve computational efficiency, some of which may not be pertinent to other BASIC operating systems running on comparable machines. Users will undoubtedly find it necessary to make modifications to the code to allow it to run on other HP equipment or in BASIC on the VAX.

\section{EXAMPLES}

The following examples demonstrate the utility of the current algorithm for application in situ and provide insight into different situations that might arise when reshading equispaced arrays with failed elements. If optimal reshading can restore the array's original design sidelobe level by slightly increasing the mainlobe beamwidth, then we say that the optimal reshading has been effective. Optimal reshading is effective in many common element-failure situations. When the array is severely degraded, optimal reshading is less effective but is still useful in reducing the negative impact of element failures. These examples demonstrate that the effectiveness of reshading depends upon the number of element failures, as well as the location of the failed elements within the array.

Missing elements are modeled by zeroing the appropriate weights. In these examples, $N$ refers to the number of intact array elements, $M$ is the number of beam pattern samples, and $K_{0}$ is calculated by using the equation in an earlier footnote. We define the mainlobe width to be twice $K_{0}$ in all three examples.

\section{A. Example 1: Effective Reshading}

This example demonstrates that reshading can restore the original sidelobe level of an array response by slightly increasing the mainlobe beamwidth. In a 25 -element equi- spaced array, originally designed for $-30-\mathrm{dB}$ sidelobes, elements 2 and 4 have failed. Therefore, $N=23, M=128$, and $K_{0}=0.6877$. We first keep the mainlobe width fixed and allow the sidelobe level to rise. See Fig. 1. The peak sidelobe level has risen to $-26.86 \mathrm{~dB}$ below the mainlobe, and the mainlobe width is unchanged. If the sidelobe level after reshading is too high, an alternative to discarding or repairing the array is to broaden the mainlobe beamwidth. In Fig. $2, K_{0}$ is increased to 0.775 and the peak sidelobe level diminishes to $-30.04 \mathrm{~dB}$ below the mainlobe. A trade-off must always be made between an enlarged mainlobe beamwidth and an acceptable peak sidelobe level. In this case the mainlobe was increased 12.7 percent in order to recover the original sidelobe level. Execution times on the HP9836C are between 1 and 2 min for Figs. 1 and 2.

\section{B. Example 2: Moderately Effective Reshading}

This example is taken from Streit and Nuttall [1]. Because of the improvements detailed in Section III, above, the current algorithm runs faster on the HP9836C than on the VAX 11/ 780 , although the floating point multiply time on the HP in its basic configuration is roughly 200 times slower than on the VAX.

Consider a linear array with 50 equispaced elements, initially designed for peak sidelobes of $-30 \mathrm{~dB}$ relative to the mainlobe. Fig. 3 shows the classical Dolph-Chebyshev beam pattern with $-30-\mathrm{dB}$ sidelobes throughout the minimization range $\left[K_{0},(2 \pi / D)-K_{0}\right]$. This was computed using the current algorithm in $6.11 \mathrm{~min}$. (This ideal case could have been computed analytically.)

Now we suppose that five elements, 7, 22, 40, 43, 50, of the array have failed. The optimal response after reshading the array is shown in Fig. 4. The peak sidelobe level has risen to $-25.51 \mathrm{~dB}$, but we have maintained mainlobe beamwidth and retained full steering capability. In this example $N=45$ and $M=128$.

This example (Fig. 4) took 7.47 minutes on the HP9836C and required 292 simplex iterations. The algorithm of Streit and Nuttall required $38.4 \mathrm{~min}$ and 402 iterations on the VAX.

Recovery of the original sidelobe level is possible (Fig. 5). The mainlobe beamwidth must be increased by the large factor 257.6 percent $\left(K_{0}=0.871\right)$ and the execution of this task takes $8.98 \mathrm{~min}$ and requires 351 iterations. The constraint that all the weights lie between 0 and 1 is used. It is necessary to use the constraint in this instance because otherwise a dislocation of the maximum response from $k=0$ results. This dislocation is due to the presence of too many negatively weighted elements.

\section{Example 3: A Severely Degraded Array}

This example shows that, for severely degraded arrays, recovery of the original sidelobe level may not be possible by increasing the mainlobe beamwidth, even after optimal reshading. Consequently, control of the level of the first sidelobe must be relinquished in order to gain control of the level of the remaining sidelobes.

Consider a 25-element array with elements 11 and 14 failed. 

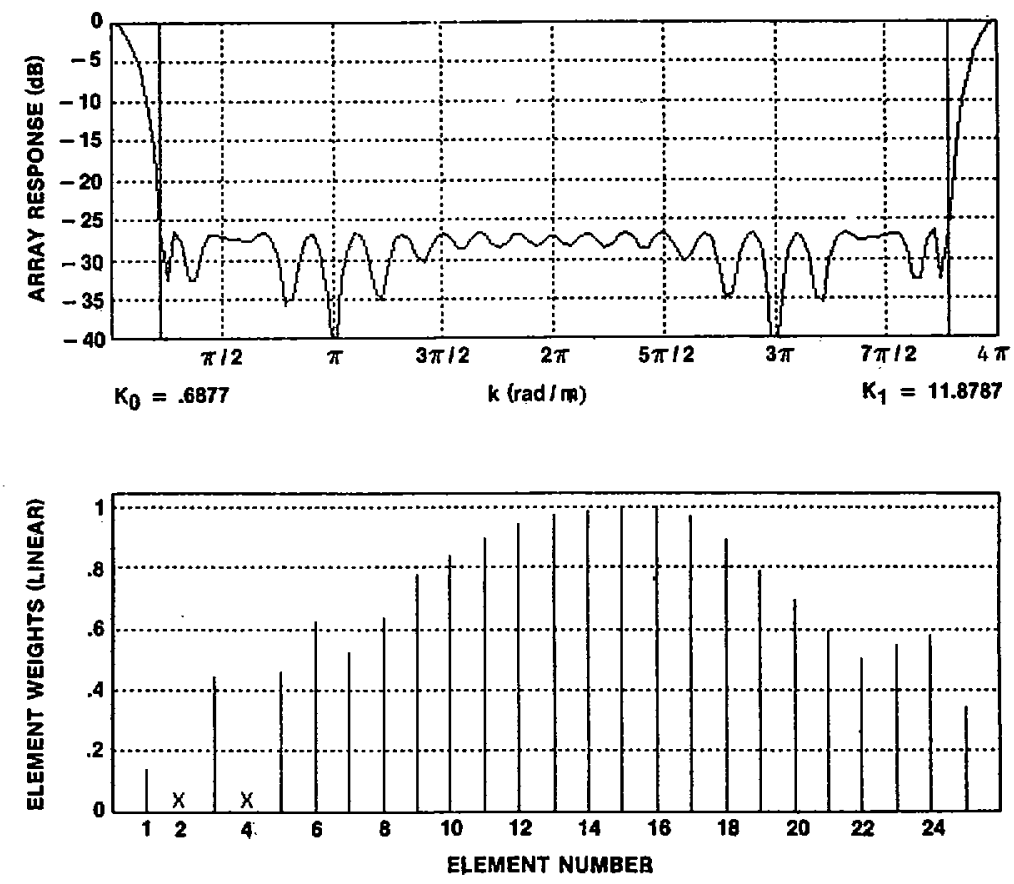

Fig. 1. Optimized array response and normalized weights for 25 elements with elements 2 and 4 missing.
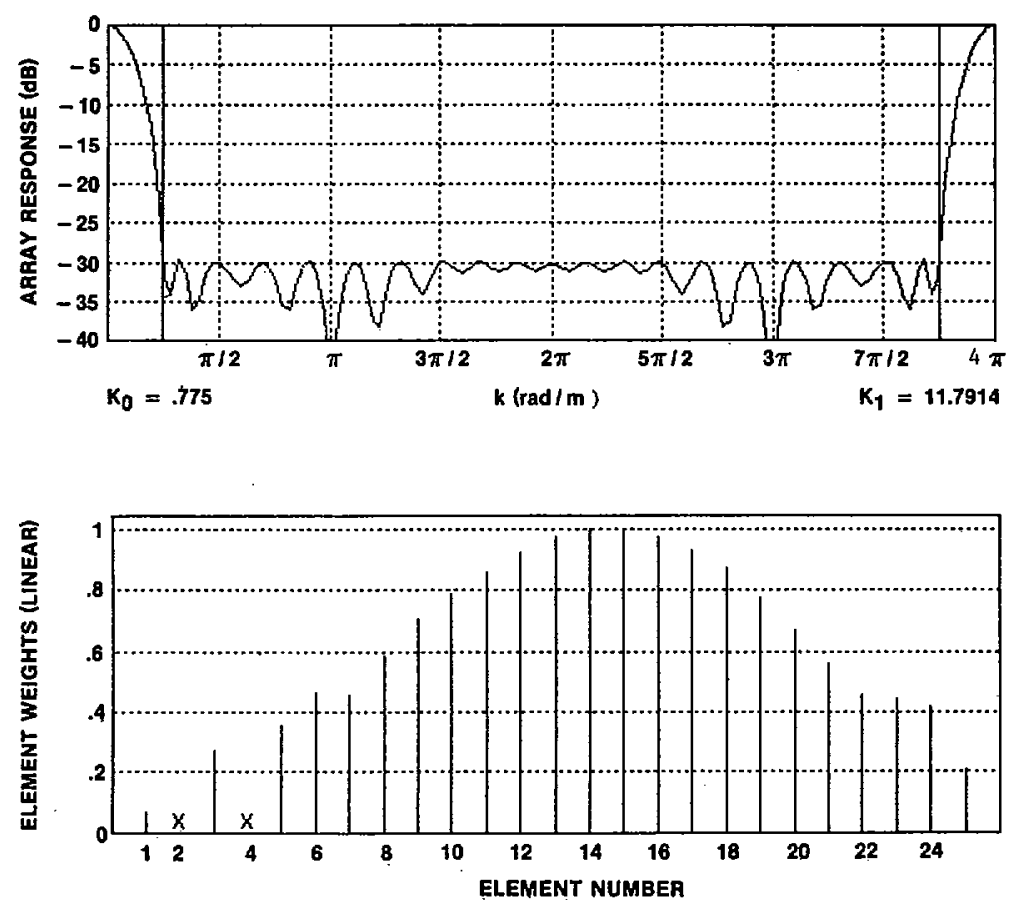

Fig. 2. Array response and normalized weights for Example 1 with $K_{0}=$ 0.775 .

The original sidelobe level is $-30 \mathrm{~dB}$. Here $N=23, M=$ 128 , and $K_{0}=0.6877$. Fig. 6 shows the algorithm's optimal response to this configuration. It is a significant observation that, in this case, small perturbations of $K_{0}$ will not affect the level of the sidelobes. Only when the first sidelobe is incorporated into the mainlobe beamwidth $\left(K_{0}=1.27\right)$ does the level of the remaining sidelobes return to the original desired value (see Fig. 7). It is apparent that decreasing the minimization interval by moving $K_{0}$ far enough to the right will improve the approximation, but one must give up control of the first sidelobe to reduce the others to acceptable levels. The net effect of losing two elements so close to the center is that negligible emphasis is placed on the remaining center elements (12 and 13) and the rest of the aperture is reshaded as if it were two separate arrays.

This situation cannot be overcome by using different 

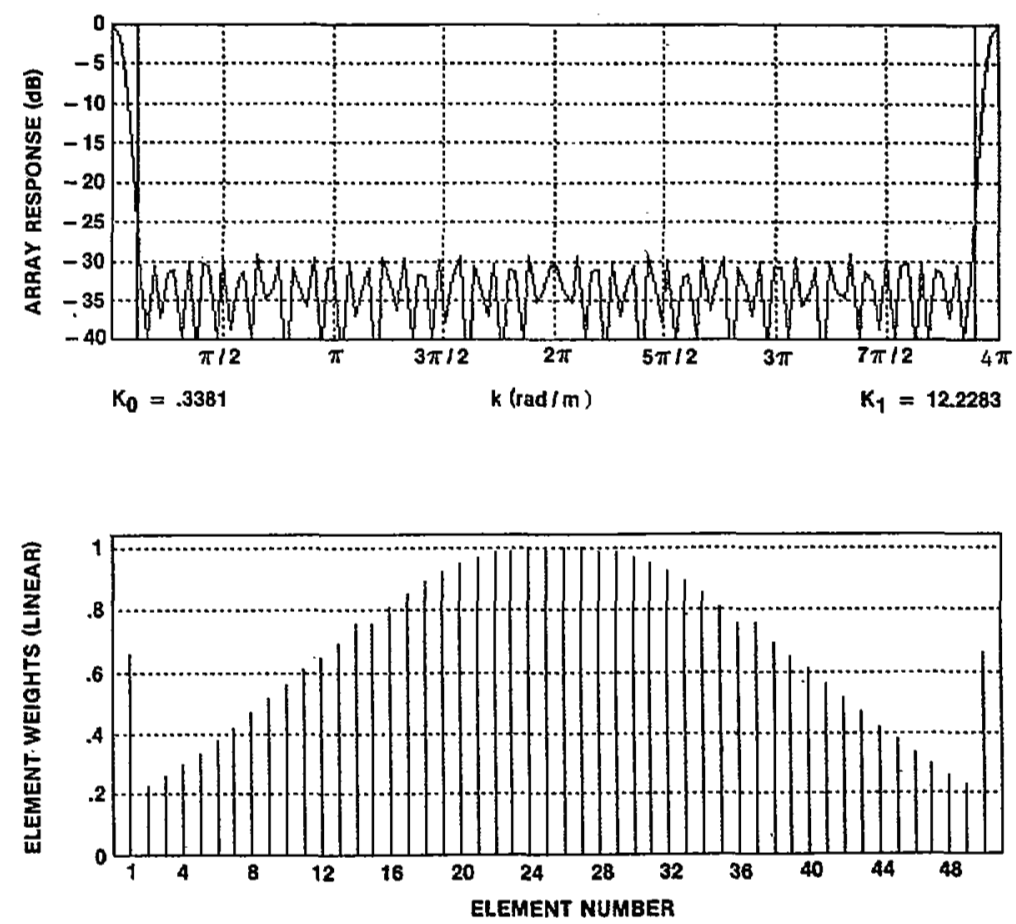

Fig. 3. Classical Dolph-Chebyshev array response and normalized weights for $N=50$ and $-30-\mathrm{dB}$ sidelobes.
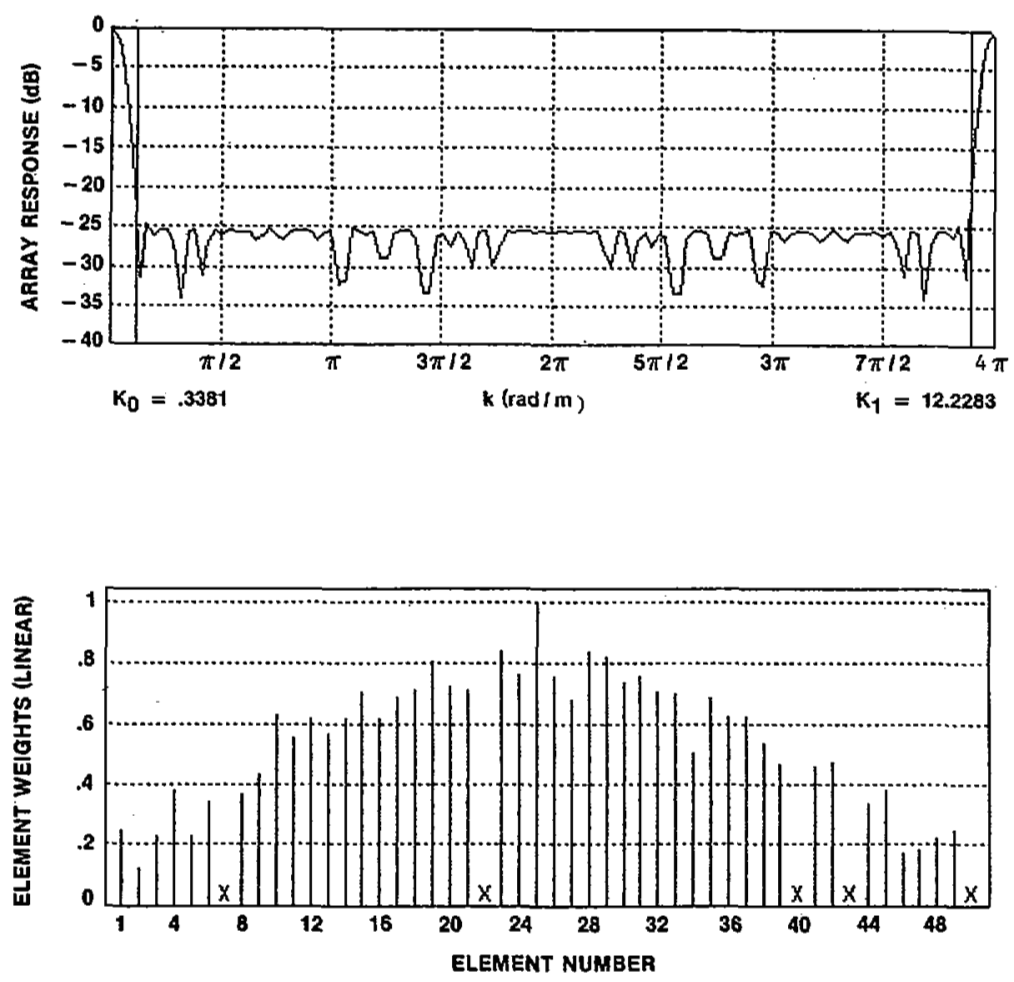

Fig. 4. Optimized array response and normalized weights for 50 elements with elements $7,22,40,43$, and 50 failed.

weights. The optimal property of the array problem formulation and solution tells us that no weights exist which can suppress all the sidelobes below a certain level. Thus this array has lost too many elements and performance cannot be restored to its original design levels merely by reshading.

We have chosen to relinquish control of the first sidelobe to gain control of the level of the remaining sidelobes. We pick the first sidelobe merely for ease of implementation; modification of the algorithm to forfeit control of a different sidelobe could also have been done. The need to relinquish control of the first sidelobe level has only appeared in cases of severe array degradation due to element losses. 

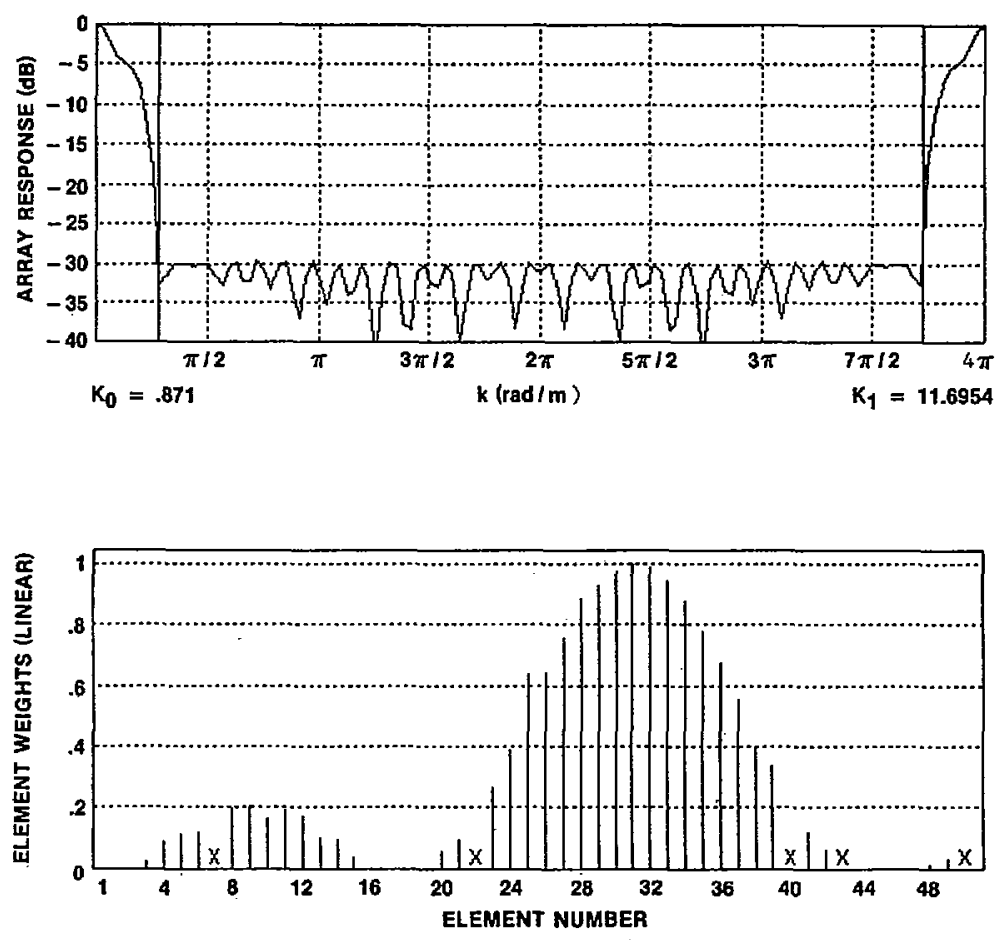

Fig. 5. Recovery of original sidelobe level, Example 2 with $K_{0}=0.871$.
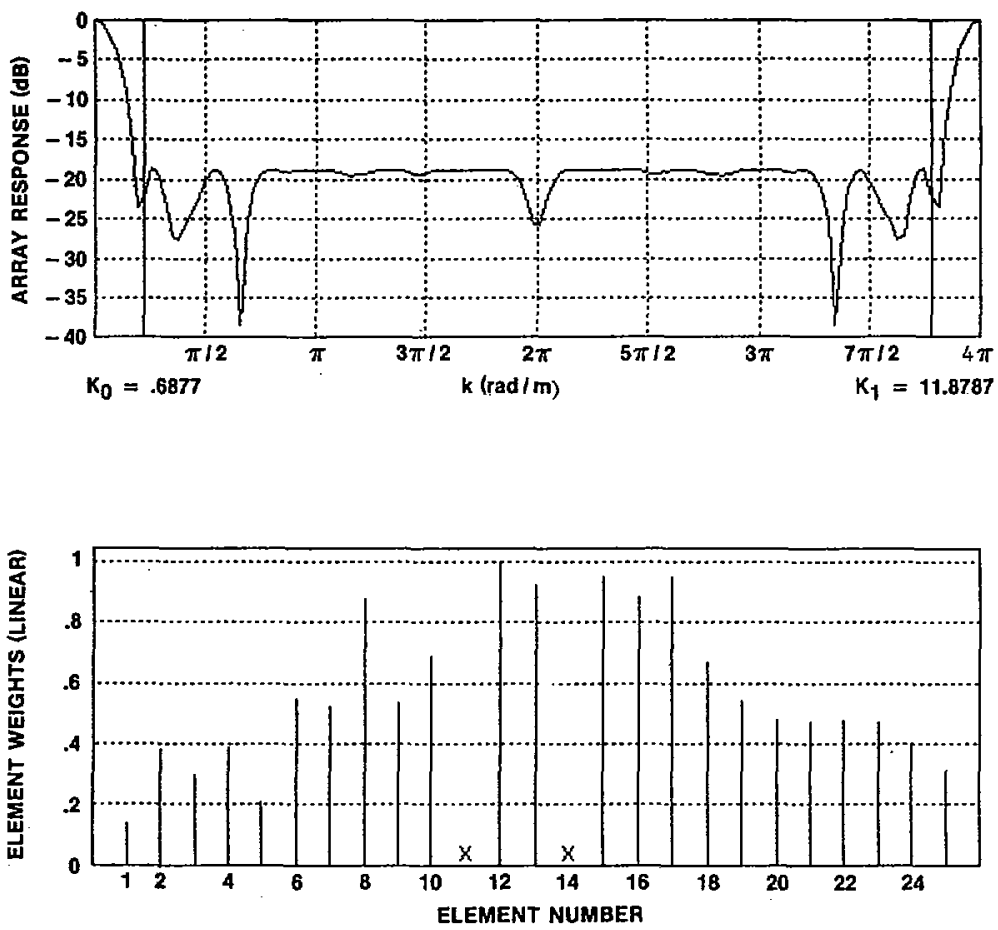

Fig. 6. Optimal array response and normalized weights for 25 elements with elements 11 and 14 failed.

\section{ConClusions}

Arrays that have failed elements can be reshaded to obtain optimal array response functions. Optimal reshading is effective in many common element-failure situations. When the array is severely degraded, reshading is less effective, but still can be used to reduce the negative impact of element failures.
Optimal reshading can be accomplished in situ, quickly and reliably, on portable microcomputers using the algorithm described here. Arrays with 25 elements routinely run in less than $2 \mathrm{~min}$ and computation time for a 50-element array is less than $10 \mathrm{~min}$. The algorithm can be applied to arrays of evenly or unevenly spaced linear geometry.

The above examples (and others) support the generally 

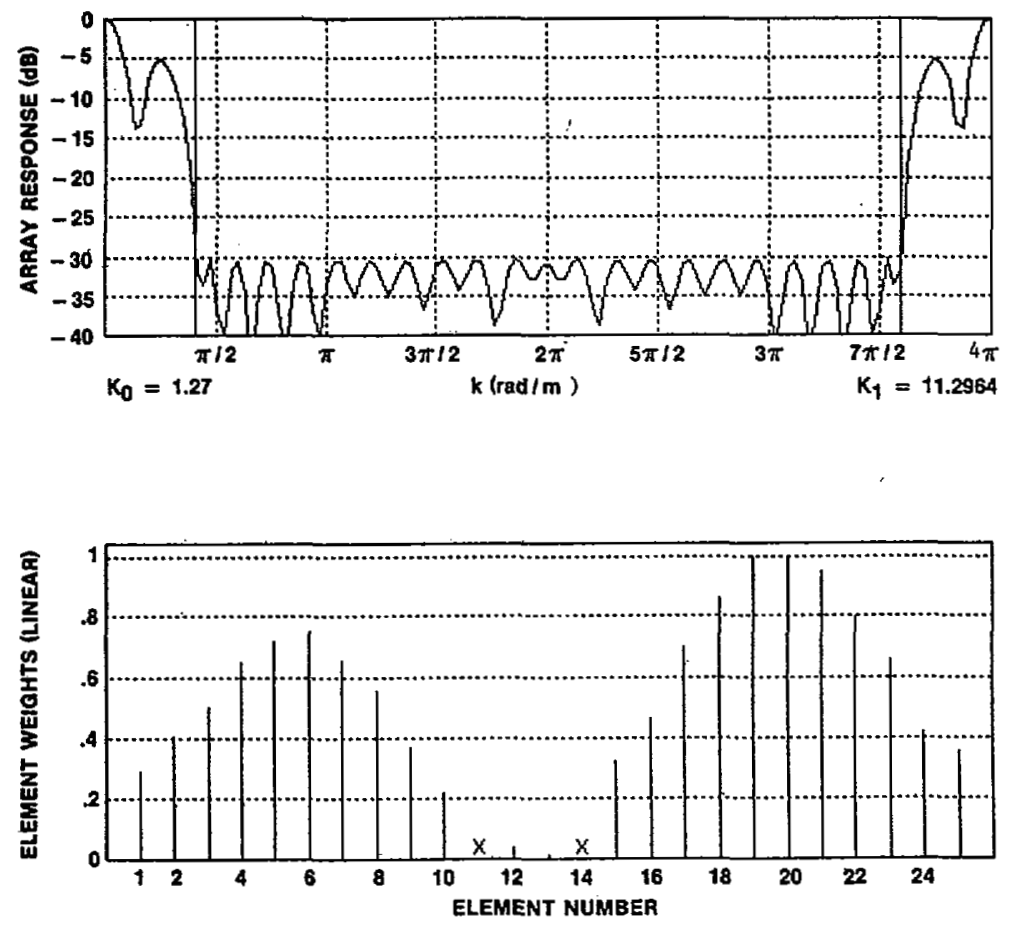

Fig. 7. Recovery of original sidelobe level, Example 3 with $K_{0}=1.27$.

accepted notion that failure of near-center elements is more detrimental to the array response than failure of near-edge elements.

Another application of Algorithm 635 is to arrays of planar and arbitrary three-dimensional geometry. Computation times for these more general arrays probably will depend upon $N$ (number of sensors) and $M$ ( number of beam pattern samples) in the same manner as for linear arrays.

\section{REFERENCES}

[1] R. L. Streit and A. H. Nuttall, "A general Chebyshev complex function approximation procedure and an application to beamforming," J. Acoust. Soc. Amer., vol. 72, pp. 181-189, 1982.

[2] I. Barrodale and C. Philips, "Solution of overdetermined systems of linear equations in the Chebyshev norm," Algorithm 495, ACM Trans. Math. Software, vol. 1, pp. 264-270, 1975.

[3] R. L. Streit, "Solution of systems of complex linear equations in the $\mathrm{L}_{\infty}$ norm with constraints on the unknowns," Soc. Indus. Appl. Math., vol. 7, no. 1, pp. 132-149, 1986.

[4] R. L. Streit, "An algorithm for the solution of systems of coriplex linear equations in the $\mathrm{L}_{\infty}$ norm with constraints on the unknowns," Algorithm 635, ACM Trans. Math. Software, vol. 11, no. 3, pp. 242-249, 1985.

[5] J. T. Lewis and R. L. Streit, "Real excitation coefficients suffice for sidelobe control in a linear array," IEEE Trans. Antennas Propagat., vol. AP-30, pp. 1262-1263, 1982; also Naval Underwater Systems Center, New London, CT, Tech. Memo. 811114, Aug. 17, 1981.

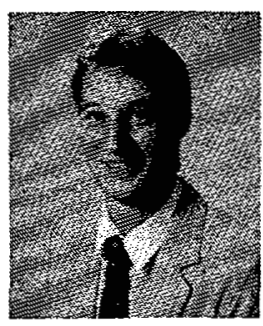

Michael S. Sherrill was born on March 8, 1961, in Dover, DE. He received the B.S. degree in electrical engineering from the University of Delaware, Newark, in 1983.

He joined the staff of the Naval Underwater Systems Center, New London, CT, upon graduation. He is interested in mathematical modeling of physical systems and structured programming. Currently, he is responsible for a data acquisition and processing system for towed arrays.

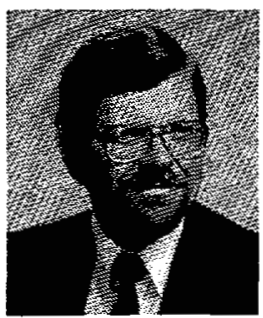

Roy L. Streit (SM'84) was born in Guthrie, OK, on October 14, 1947. He received the B.A. degree (with honors) in mathematics and physics from East Texas State University, Commerce, in 1968, the M.A. degree in mathematics from the University of Missouri, Columbia, in 1970, and the Ph.D. degree in mathematics from the University of Rhode Island, Kingston, in 1978.

He is currently an Adjunct Associate Professor of the Department of Mathematics, University of Rhode Island. He was a Visiting Scholar in the Department of Operations Research, Stanford University, Stanford, CA, during 1981-1982. He joined the staff of the Naval Underwater Systems Center, New London, CT (then the Underwater Sound Laboratory), in 1970. $\mathrm{He}$ is an Applied Mathematician and has published work in several areas, including antenna design, complex function approximation theory and methods, and semi-infinite programming. He is currently conducting research in towed array design and hidden Markov models. 